\title{
Effect of Mesenchymal Stem Cells Associated to Matrixen on the Erectile Function in the Rat Model with Bilateral Cavernous Nerve Crushing Injury
}

\author{
Su Jin Kim, Sang Hoon Park, Young Chul Sung, Sae Woong Kim \\ Department of Urology, Catholic University of Korea, College of Medicine (SJK, SWK) and Division of \\ Molecular and Life Science, Integrative Bioscience and Biotechnology, WCU, Pohang University of Science \\ and Technology (POSTECH) (SHP, YCS), Pohang, Republic of Korea
}

\section{ABSTRACT}

Objectives: To evaluate the effect of mesenchymal stem cells (MSCs) and MSCs mixed with Matrixen as a cell carrier on the erectile dysfunction caused by bilateral cavernous nerve crushing injury.

Materials and Methods: White male Sprague-Dawley rats were divided into 4 groups: sham-operated control group $(n=5)$, bilateral cavernous nerve crushing group (BCNC group, $n=10)$, BCNC administered with MSCs group ( $n=10,1 \times 106$ in $20 \mu \mathrm{L})$, BCNC administered with Matrixen group ( $\mathrm{n}=10.1 \times 106$ in $20 \mu \mathrm{L}$ ), BCNC administered with MSCs/Matrixen group ( $\mathrm{n}=10.1 \times 106$ in $20 \mu \mathrm{L})$. After functional assessment at 4 weeks, major pelvic ganglion (MPG) and penile tissue were collected. Immunofluorescent staining of MPG was performed with PKH26 and Tuj1. Western blot analysis of endothelial nitric oxide synthase (eNOS) and neuronal nitric oxide synthase (nNOS) were done in corpus cavernosum.

Results: ICP/MAP ratios of BCNC with MSCs and MSCs/Matrixen groups were significantly increased compared with BCNC and BCNC with Matrixen group. Moreover, ICP/ MAP ratios of MSCs/Matrixen group were significantly increased compared with BCNC with MSCs group. In MPG, the more implantation of MSCs and increased expression of nerve cells were observed in MSCs/Matrixen group compared with BCNC with MSCs group. Significant increase expression of eNOS and nNOS was also noted in BCNC with MSCs/Matrixen group.

Conclusion: The erectile function was more preserved in MSCs/Matrixen group compared with the administration of MSCs alone in the rats with bilateral cavernous nerve crushing injury. Therefore, we consider that the use of transplant cell carrier such as Matrixen may help the implantation of MSCs and improve the therapeutic effect of MSCs.

\section{ARTICLE INFO}

\section{Key words:}

Cavernous nerve; Erectile dysfunction; Matrixen;

Mesenchymal stem cell

Int Braz J Urol. 2012; 38: 833-41

Submitted for publication:

April 25, 2012

Accepted after revision: August 14, 2012

\section{INTRODUCTION}

Diagnosis of prostate cancer has been elevating due to the increase in prostate-specific antigen (PSA) screening. In addition, this earlier diagnosis increases the number of patients pre- senting with clinically organ-confined disease and therefore, it has led to an increase of the number of candidates for radical prostatectomy $(1,2)$. Radical prostatectomy has been associated with complications such as postoperative urinary incontinence and erectile dysfunction. Erectile dysfunction is a bothersome complaint among pa- 
tients submitted to radical prostatectomy and it is an important reason for the decrease of the quality of life in these patients. Therefore, nerve-sparing procedures have been proposed to reduce short-term and long-term functional complications such as erectile dysfunction (3-5). However, some patients submitted to radical prostatectomy with nerve-sparing technique have been experiencing erectile dysfunction and need other treatments to improve their symptoms (6).

Mesenchymal derived stem cells (MSCs) from bone marrow seems to be one of the attractive treatments for tissue regeneration and engineering due to their differentiation potentials into various adult tissues, including endothelial cell, smooth muscle and nerve (7). Therefore, there have been several studies using MSCs and the treatment effect of MSCs has been studied in the animal model of erectile dysfunction caused by cavernous nerve injury. As a result, stem cell therapy may be one of the treatment options for the patients that lost their potency after radical prostatectomy. However, some difficulties appeared in stem cell therapy in spite of their therapeutic effect. For example, stem cells were easily spread-out to the adjacent tissue after stem cell injection into the target tissue according to the report by Lee et al. (8). This may cause unexpected, unfavorable events and moreover, we are not able to get anticipating results from the stem cell therapy, neither. To overcome this weakness, some investigators used polymers to promote the retention of stem cells in the targeted tissue (9-11).

Among various biomaterials, Matrixen is a collagen based biocompatible polymer and it has been used widely as scaffolds in medical field. In addition, Matrixen is easy to handle depending on the investigator's purpose due to its characteristics of thermo-dependant sol-to-gel transition. Therefore, we used Matrixen to enhance the implantation ability of MSCs and compared the effect of MSCs and MSCs mixed with Matrixen in rats with cavernous nerve crushing injury.

\section{MATERIALS AND METHODS}

\section{Animals}

White male Sprague-Dawley rats aged 8 weeks with weight distribution of 300-350 gm were purchased from Saemataco Inc. (Osan, Korea). Then, the rats were divided into 5 groups: the control group $(n=5)$, the bilateral cavernous nerve crushing group (BCNC group, $n=10$ ), the administration of mesenchymal stem cells following the bilateral cavernous nerve crushing (BCNC with MSCs group, $n=10$ ), the administration of Matrixen following the bilateral cavernous nerve crushing (BCNC with Matrixen group, $\mathrm{n}=$ 10), and the administration with MSCs mixed with Matrixen following the bilateral cavernous nerve crushing (BCNC with MSCs/Matrixen group, $\mathrm{n}=$ 10). The experimental protocol was approved by the Catholic University Animal Ethics Committee (CUMC-2009-0045-01).

\section{Preparation of MSCs}

For preparation of rat bone marrow-derived MSCs (rBM-MSCs), bone marrow cells were collected from the femurs and tibias of 3-4 week-old Sprague-Dawley rats by flushing the respective tissues with Hank's balanced salt solution (HBSS, WelGENE, Korea) containing 2\% fetal bovine serum (FBS; Hyclone, USA). After red blood cells were removed, bone marrow cells were filtered through a $40 \mu \mathrm{m}$ cell strainer (BD Bioscience, San Jose, CA) and separated using Ficoll density gradient centrifugation. Isolated bone marrow cells were re-suspended and cultured in Dulbecco's Modified Eagle Medium (DMEM, 1000 $\mathrm{mg} / \mathrm{L}$ glucose; WelGENE) with 20\% FBS, $100 \mathrm{U} / \mathrm{mL}$ penicillin and $100 \mathrm{~g} / \mathrm{mL}$ of streptomycin (Invitrogen, Carlsbad, CA) for about 10 days until colonies formed. Colonies were harvested and used for subsequent experiments as MSCs. For animal experiments, ex-vivo expanded MSCs (less than passage 5) were seeded into a $70 \mathrm{~mm}$ flask at an initial density of $1.4 \times 106$ cells and incubated overnight at $37^{\circ} \mathrm{C}$. Cells were then trypsinized, washed with PBS, and injected into the rats (1x106 cells/rat).

Bilateral cavernous nerve crushing and administration of MSCs or MSCs mixed with Matrixen

Tiletamine (Zoletil) $0.2 \mathrm{~mL}$ was injected intraperitoneally to anesthetize the animals. A lower midline incision was made and the prostate gland was exposed. After identifying the major pelvis ganglion (MPG) on the lateral side to bi- 
lateral prostates, the cavernous nerves, tracking posterolaterally, were identified and isolated. In control group (sham surgery), there was no further surgical manipulation. In the remaining groups the cavernous nerves were isolated and a crush injury was induced using a hemostat clamp for 2 min. In the BCNC group, the abdomen was closed after the bilateral cavernous nerve crushing. In the BCNC with MSCs group, MSCs (1x106 in $20 \mu \mathrm{L}$ ) were administered to the MPG, Matrixen $(20 \mu \mathrm{L})$ was administered to the MPG, and MSC $(1 \times 106$ in $20 \mu \mathrm{L})$ mixed with Matrixen (Bioland, Cheongwon, Chunbuk, KOREA) administered to the MPG in BCNC with MSCs/Matrixen group after bilateral cavernous nerve crushing (Figure-1).

Figure 1 - The gross finding of MSCs plus collagen based cell carrier after immediate administration into MPG. A: MPG of normal rats, B: Gel formation of collagen based cell carrier contacting of MSCs after implantation, MPG: major pelvic ganglion, MSCs: mesenchymal stem cells.
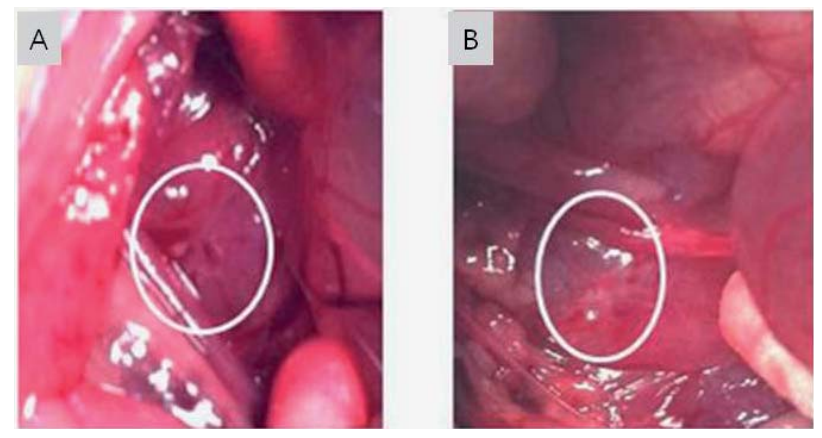

Erectile functional assessment

The rats were anaesthetized with an intraperitoneal injection of $0.2 \mathrm{~mL}$ Tiletamine (Zoletil ${ }^{\circledR}$. With the rat in the supine position, the penis was dissected and the corpus cavernosum and crus of the penis were exposed. A low, midline abdominal incision was made to access the pelvis, and the MPG lateral to the right prostate was exposed. For the measurement of the intracavernosal pressure (ICP), a heparinized 23G butterfly needle was inserted in the corpus cavernosum of penile proximal portion after penile skin was degloved and the corpus cavernosum identified. For the measurement of mean arterial pressure (MAP), a PE-50 tube was inserted into the carotid artery.
Then a bipolar electrical stimulator was placed on the ganglion to stimulate the cavernous nerve for 50 seconds at $1.5 \mathrm{~mA}, 20 \mathrm{~Hz}$, pulse width $0.2 \mathrm{~ms}$. The cavernous nerve stimulation was conducted at least 3 times and the interval between stimulations was maintained for over 10 minutes. At the completion of functional analysis, the MPG and penis were excised for histopathology.

\section{Immunofluoescent staining of MSCs in MPG}

Immediately following ICP measurement, the ganglion was removed. The tissue was then snap frozen using 2-methylbutane pre-cooled in liquid nitrogen. Cryostat sections of the ganglion embedded in tissue-Tek OCT were obtained. From each ganglion, consecutive sections $(5 \mu \mathrm{m})$ were collected on 4-5 slides, and thus each contained a similar collection of 10-15 serial sections from the same animal.

Ganglion sections were washed three times for 5 min with PBS and to avoid nonspecific antibody binding; they were then incubated for 60 min with 2\% normal goat serum (Chemicon International, Temecula, CA) containing $0.1 \%$ Triton $\mathrm{X}-100$. To evaluate neuronal differentiation of MSCs, the samples were incubated with anti-beta-tublin III (Tuj1) (diluted to 1:200; Abcam, Cambridge, UK) overnight at $4{ }^{\circ} \mathrm{C}$ in a humidified chamber. Sections were incubated with the secondary antibody (Alexa Fluor 488 goat anti-rabbit IgG; Invitrogen) for 2 hours in the dark in a humidified chamber at room temperature. After a washing (3 times, $10 \mathrm{~min}$. each) with PBS, the nuclei were counterstained with 4,6-diamino-2-phenylindole dihydrochloride (DAPI;vector Labs, Burlingame, CA). Immunofluorescence was visualized using an Olympus BX51 fluorescence microscope.

eNOS and nNOS protein expression tests: Western blot

The corpus cavernosum was obtained from all rats and homogenized individually in a buffer solution of $0.32 \mathrm{M}$ sucrose, 0.2M Hepes, (pH 7.4), $1 \mathrm{mM}$ ethylenediaminetetraacetic acid (EDTA), $1 \mathrm{mM}$ dithiothreitol (DTT), $10 \mu \mathrm{g} / \mathrm{mL}$ leupeptin, 2 $\mu \mathrm{g} / \mathrm{mL}$ aprotinin, $1 \mu \mathrm{g} / \mathrm{mL}$ pepstatin, $10 \mu \mathrm{g} / \mathrm{mL}$ trypsin inhibitor and $1 \mathrm{mM}$ phenylmethylsulfonyl 
fluoride (PMSF). The homogenized buffer solution was placed on ice for $15 \mathrm{~min}$ and centrifuged at 4 ${ }^{\circ} \mathrm{C}$ for 13,000 rpm for 15 minutes. The supernatant solution was separated. The separated solution was utilized in the bovine serum albumin. $30 \mu \mathrm{g}$ of the quantitative protein was denatured at $95{ }^{\circ} \mathrm{C}$ for 5 min. and electrophoresis was performed on a $12 \%$ discontinuous sodium dodecylsulfate (SDSPAGE)-polyacrylamide gel. The proteins were then electroblotted onto a $0.2 \mu \mathrm{m}$ polyvinylidenedifluoride (PVDF, Amershambioscience, USA) membrane for 150 minutes at $25 \mathrm{~V}$. The membranes were reacted with blocking buffer (5\% skim milk in TBS-T buffer) for 30 minutes at the ambient temperature. The eNOS and nNOS (BD Biosciences, USA) antibodies were added for 2 hours, and the membrane was washed 3 times using TTBS at intervals of $10 \mathrm{~min}$. The secondary antibodies, anti-mouse IgG-HRP and anti-goat IgG-HRP (1:2000 dilution) (Zymed Laboratories Laboratories, USA) were added at the ambient temperature for 1 hours and the membrane was washed again with TTBS for 6 times with an interval of 5 minutes between each washing. Chemiluminescence was detected using ECL Western blotting detection reagents. Densitometric assessment of the bands on the autoradiogram was performed using Bio1D software (version 97; Vilber Lourmat, France).

\section{Statistical analysis}

All measurements were expressed as mean \pm standard deviation (SD). Statistical analysis was obtained via Sigma stat for Windows. An inter-group comparison was made with the use of Neuman-Keuls multiple comparison test. The cut-off value of statistical significance was $\mathrm{P}<0.05$.

\section{RESULTS}

\section{Erectile functional assessment}

The erectile function was assessed by tracing the ICP under cavernous nerve electrical stimulation at 4 weeks after operation. The analysis is presented in ICP/MAP ratios (Figure- 2). The ICP/MAP ratios in the control group were 0.83 \pm 0.02 , which was significantly higher compared with all other groups $(\mathrm{P}<0.05)$. BCNC group sho- wed significantly decreased ICP/MAP ratios $0.23 \pm$ $0.04(\mathrm{P}<0.05)$. The ICP/MAP ratios $(0.21 \pm 0.08)$ of BCNC with Matrixen group was significantly decreased compared with control group $(\mathrm{P}<0.05)$. The ICP/MAP ratios of BCNC with MSCs group $(0.49 \pm 0.1)$ and BCNC with MSCs/Matrixen group $(0.65 \pm 0.03)$ were significantly higher compared with $\mathrm{BCNC}$ group $(0.23 \pm 0.04)(\mathrm{P}<0.05)$. Moreover the the ICP/MAP ratios of BCNC with MSCs/ Matrixen group $(0.67 \pm 0.09)$ was significantly higher compared with BCNC with MSCs group (0.49 $\pm 0.1)(\mathrm{P}<0.05)$.

Expression of MSCs in MPG using immunofluoescent staining

MPG in BCNC group showed decreased expression of neuronal cells compared with that of control group. The expression of neuronal cells was increased in BCNC with MSCs and BCNC with MSC/Matrixen groups compared with BCNC group. In the MCSs/Matrixen group, increased implantation of MSCs was observed in MPGs compared with BCNC with MSCs group (Figure-3).

\section{Quantification of eNOS and nNOS proteins}

Decreased expressions of eNOS and nNOS were observed in the BCNC and BCNC with Matrixen groups than the control group $(\mathrm{P}<0.05)$. The increase expressions of the eNOS and nNOS was observed in the BCNC with MSC and BCNC with MSCs/Matrixen groups compared with BCNC group $(\mathrm{P}<0.05)$. The significant increase expression of the eNOS and nNOS was observed in BCNC with MSC/Matrixen group compared with BCNC with MSCs group $(\mathrm{P}<0.05)$ (Figure-4).

\section{DISCUSSION}

We observed that the administration of MSCs into major pelvic ganglion (MPG) showed the preservation of erectile function after bilateral cavernous nerve crushing injury. Moreover, the implantation of MSCs was enhanced in the MPG with the help of collagen based cell carrier, Matrixen, and the improvements of functional and histological results were noted in this study.

Animal model induced by bilateral cavernous nerve crushing injury has been widely used 
Figure 2 - Erectile functional assessment under cavernous nerve stimulation (CNS) at week 4 in control (A); bilateral cavernous nerve (BCNC) crushing (B); BCNC with Matrixen (C); BCNC with mesenchymal stem cells (MSCs) (D); BCNC with MSCs mixed with Matrixen group (E). The voltage dependent erectile response to CNS represented by the ratios of intracavernous pressure (ICP) / mean arterial pressure (MAP) (F). ${ }^{*} \mathrm{P}<0.05$ compared with the control group, ${ }^{*} \mathrm{P}<0.05$ compared with BCNC group, †P $<0.05$ compared with BCNC injected with MSCs group.
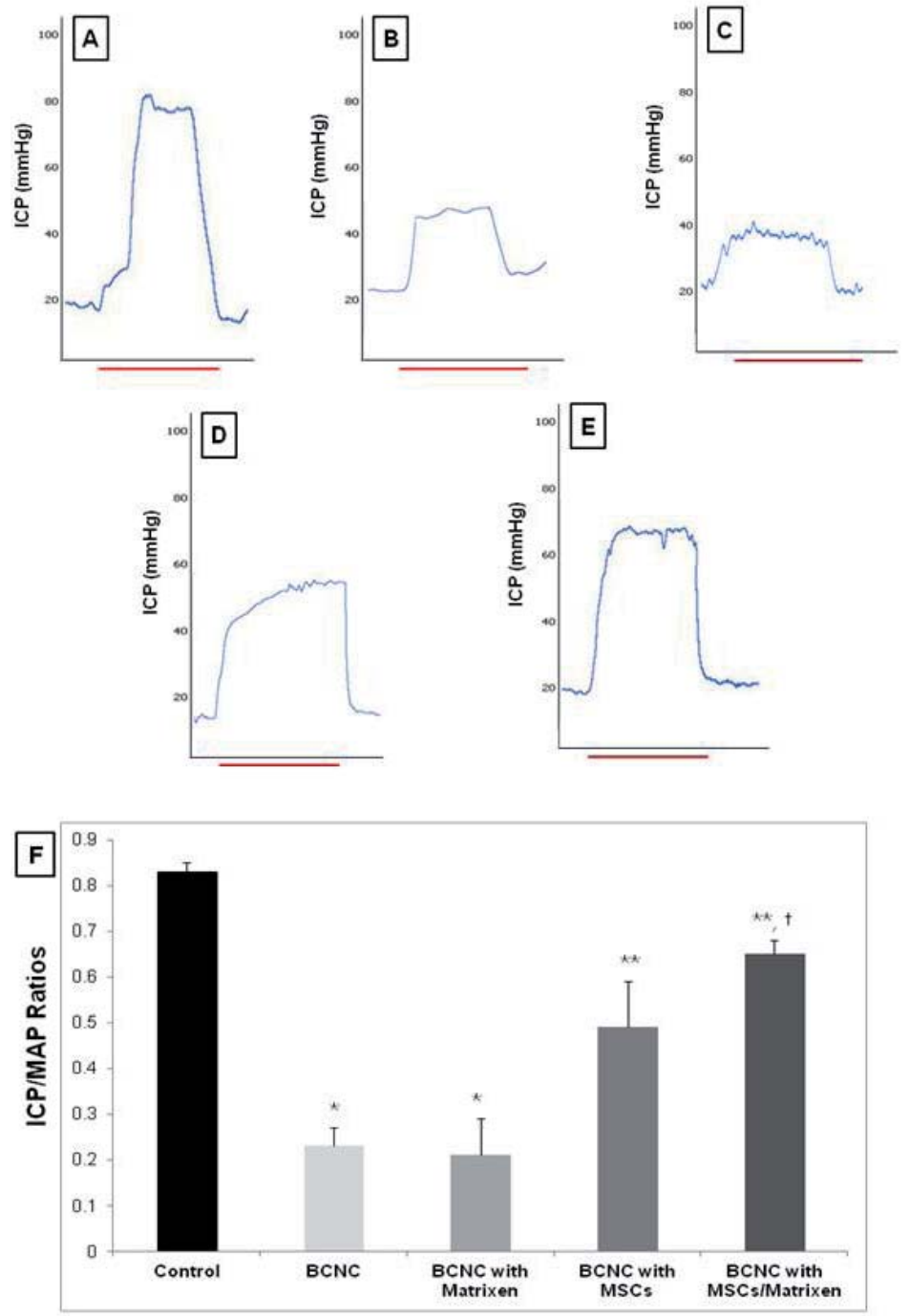

because this type of injury was designed to mimic the partial nerve damage that occurs during nerve-sparing radical prostatectomy through mechanical nerve stretch (11). And several studies were performed about the periodic changes of MPG in response to the cavernous nerve injury in the animals with erectile dysfunction induced by cavernous nerve injury $(12,13)$. For example, Palma et al. (12) observed the sprouting of injured postganglionic axons in MPG of adult male rats at the first week after unilateral cavernous nerve transaction or crushing. And the recovery of cavernous nerve probably partly depends on sprouting of the remaining neural tissue after adverse manipulation. However, clinically, the recovery of erectile function cannot be observed in all patients who are 
Figure 3 - The expression of mesenchymal derived stem cells (MSCs) in MPG at 4 weeks in control control (A); bilateral cavernous nerve (BCNC) crushing (B); BCNC with Matrixen (C); BCNC with mesenchymal stem cells (MSCs) (D); BCNC with MSCs mixed with Matrixen group (E). (x 400, blue:DAPI, green:tuj1, red: PKH26)


supposed to experience the partial nerve damage that occurs during nerve-sparing radical prostatectomy (6). As a result, stem cell therapy has been introduced as one of therapeutic options to help the preservation and restoration of erectile function in these patients (4). Therefore, we administered MSCs to MPG to evaluate the influence on the recovery of neurogenic erectile dysfunction by bilateral cavernous crushing injury. Moreover, we evaluated the role of Matrixen as a stem cell carrier to promote the implantation ability of MSCs in MPG by administration of MSCs mixed with Matrixen.

At 4 weeks after injury, ICP/MAP ratios in BCNC with MSCs and BCNC with MSCs mixed with Matrixen were significantly elevated compared with BCNC group. Even more elevated ICP/ MAP ratio was observed in the BCNC with MSCs/ Matrixen group than in the BCNC with MSCs group. This result supposed that more implantation of MSCs was possible in MPG, since Matrixen prevented the spread-out of MSCs to the adjacent, unexpected area form the MPG. And this result was identified with increased expression of stem cell in MPG in the BCNC with MSCs/Matrixen group compared with the BCNC with MSCs group from the immunofluorescent staining results.

Currently, MSCs suspended in cell culture medium are administered and therefore, there may be a limitation in the cell retention and transplant survival $(15,16)$. There have been several studies to promote cell survival and retention, for example, the use of biomaterial scaffolds, which provide a microporous framework to improve cell survival $(16,17) . \mathrm{Xu}$ et al. (9) reported treatment effect of the muscle derived stem cell (MDSC) mixed with fibrin glue on the restoration of urethral sphincter function resulted from the pudendal nerve transaction rat model. They observed an obvious increase of the thickness of muscle mass and of the muscle/collagen ratio, as well as a higher neovasculature density after MDSC mixed with fibrin glue injection. There is another study about the effect of MDSC mixed with polymer such as alginate (Alg)/polycaprolactone (PCL) in the same urethral sphincter dysfunction animal model (10). It also observed significant functional improvement compared with injection with MDSCs, alone and 
Figure 4 - Westerm blot analysis of eNOS and nNOS in corpus cavernosum at 4 weeks control, bilateral cavernous nerve crushing (BCNC), BCNC with Matrixen, BCNC with mesenchymal stem cells (MSCs), BCNC with MSCs mixed with Matrixen group (A). Densimetric analysis to $\beta$-actin of eNOS and nNOS (B).

${ }^{*} \mathrm{P}<0.05$ compared with control group. ${ }^{*} \mathrm{P}<0.05$ compared with BCNC group. $\mathrm{\dagger P}<0.05$ compared with BCNC with MSCs group.

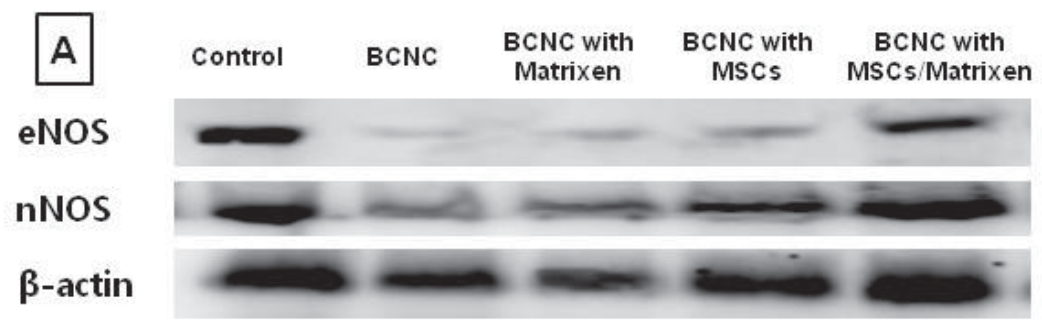

B
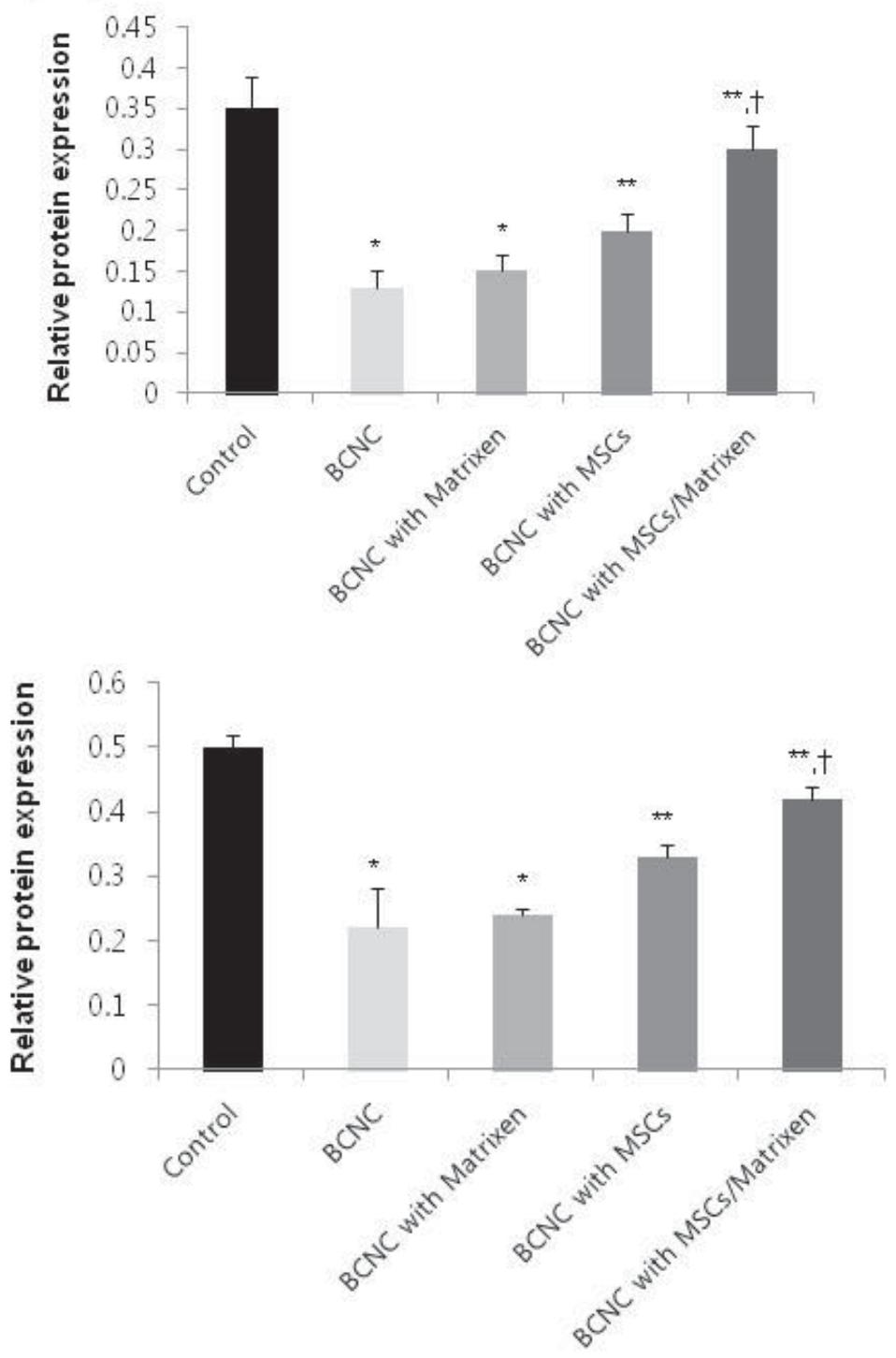
suggested that the polymer may promote the stem cell viability and bulking effect. To be clinically useful cell transplant carriers, there are several required conditions. They need to be biocompatible without toxic side effects and biodegradable. And the ideal preparation must be liquid because liquid would be shaped and administered more easily.

We used Matrixen as stem cell carrier because it is collagen based biocompatible polymer and also shows thermosensitive character. It has easily sol-to-gel transition character that depends on the temperature. Matrixen remains as a sol state at the low temperature; therefore, it can be easily mixed with MSCs. After implantation into MPG with MSCs mixed with Matrixen, we could observe the immediate sol-to-gel transition and the gel formed three-dimensional shape at the body temperature. This means that the gel formation rate is fast. If the process of gel formation is slow Matrixen spreads to form a sheet like structure $(19,20)$ and it may disturb the retention and localization of MSCs in MPG. Therefore, the better preservation of eNOS and nNOS levels in BCNC with MSCs/Matrixen group may be due to the stable immobilization of MSCs by Matrixen in MPG compared with BCNC with MSCs group. In addition, there was a study that suggested the necessity of a cell transplant carrier in other aspect. They observed that relatively large amount of MDSCs was needed for the functional improvement in the rats of intrinsic sphincter deficiency due to the spread-out of MDSCs (21). Practically, it is impossible to use a large number of MSCs for the regeneration of damaged tissue because the acceptable capacity may be limited according to the size of damaged tissue. However, the administration with Matrixen after BCNC did not show functional and molecular improvement compared with BCNC with MSC/Matrixen group. From this finding, we consider that Matrixen does not have a role in the restoration of damaged nerve tissue and its ability seems to be a simple cell carrier to enhance cell implantation.

The safety of MSCs has been well demonstrated $(22,23)$, however, there is a probable chance to occur unexpected adverse events after MSCs treatment. Thus it is important to make MSCs act on the targeted damage tissue as possible and we expect the better treatment outcome of MSCs with the help of transplant cell carrier such as Matrixen. As a result, Matrixen is supposed to enhance the implantation of MSCs and differentiate to neural cells in MPG.

\section{CONCLUSIONS}

After administration with MSCs, alone, functional and histological restoration was observed in the rats with bilateral cavernous nerve crushing injury. In addition, the effect of MSCs on recovery of erectile function might be improved by using the cell carrier such as Matrixen. The reason of this result seemed that Matrixen might prevent spread-out of MSCs after administration. Therefore, we considered that the use of transplant cell carrier such as Matrixen may help the implantation of MSCs and improve the therapeutic effect of MSCs.

\section{ACKNOWLEDGMENT}

This work was supported by the Catholic Institute of Cell Therapy in 2011.

\section{CONFLICT OF INTEREST}

None declared.

\section{REFERENCES}

1. Schröder FH, Carter HB, Wolters T, van den Bergh RC, Gosselaar C, Bangma CH, et al.: Early detection of prostate cancer in 2007. Part 1: PSA and PSA kinetics. Eur Urol. 2008; 53: 468-77.

2. Bove P, Asimakopoulos AD, Kim FJ, Vespasiani G: Laparoscopic radical prostatectomy: a review. Int Braz J Urol. 2009; 35: 125-37; discussion 137-9.

3. Walsh PC: Radical prostatectomy for localized prostate cancer provides durable cancer control with excellent quality of life: a structured debate. J Urol. 2000; 163: 1802-7.

4. Montorsi F, Salonia A, Suardi N, Gallina A, Zanni G, Briganti $A$, et al.: Improving the preservation of the urethral sphincter and neurovascular bundles during open radical retropubic prostatectomy. Eur Urol. 2005; 48: 938-45.

5. Graefen M, Walz J, Huland H: Open retropubic nerve-sparing radical prostatectomy. Eur Urol. 2006; 49: 38-48. 
6. Zippe CD, Pahlajani G: Penile rehabilitation following radical prostatectomy: role of early intervention and chronic therapy. Urol Clin North Am. 2007; 34: 601-18.

7. Deans RJ, Moseley AB: Mesenchymal stem cells: biology and potential clinical uses. Exp Hematol. 2000; 28: 875-84.

8. Lee JY, Cannon TW, Pruchnic R, Fraser M0, Huard J, Chancellor MB: The effects of periurethral muscle-derived stem cell injection on leak point pressure in a rat model of stress urinary incontinence. Int Urogynecol J Pelvic Floor Dysfunct. 2003; 14: 31-7; discussion 37.

9. $\mathrm{Xu} Y$ Y, Song YF, Lin ZX: Transplantation of muscle-derived stem cells plus biodegradable fibrin glue restores the urethral sphincter in a pudendal nerve-transected rat model. Braz J Med Biol Res. 2010; 43: 1076-83.

10. Lee HN, Lee JY, Koh JS, Kim HW, Byun SS, Lee SS, et al.: Muscle Derived Stem Cell/Alginate/Polycaprolactone/Injection Therapy in Rats with Denervated Urethral Sphincter. Korean J Urol. 2007; 48: 1296-1301.

11. Canguven 0 , Burnett $A$ : Cavernous nerve injury using rodent animal models. J Sex Med. 2008; 5: 1776-85.

12. Palma CA, Keast JR: Structural effects and potential changes in growth factor signalling in penis-projecting autonomic neurons after axotomy. BMC Neurosci. 2006; 7: 41.

13. Carrier S, Zvara P, Nunes L, Kour NW, Rehman J, Lue TF: Regeneration of nitric oxide synthase-containing nerves after cavernous nerve neurotomy in the rat. J Urol. 1995; 153: 1722-7.

14. Harraz A, Shindel AW, Lue TF: Emerging gene and stem cell therapies for the treatment of erectile dysfunction. Nat Rev Urol. 2010; 7: 143-52.
15. Müller-Ehmsen J, Whittaker P, Kloner RA, Dow JS, Sakoda T, Long TI, et al.: Survival and development of neonatal rat cardiomyocytes transplanted into adult myocardium. J Mol Cell Cardiol. 2002; 34: 107-16.

16. Reinecke H, Murry CE: Taking the death toll after cardiomyocyte grafting: a reminder of the importance of quantitative biology. J Mol Cell Cardiol. 2002; 34: 251-3.

17. Gutowska A, Jeong $B$, Jasionowski M: Injectable gels for tissue engineering. Anat Rec. 2001; 263: 342-9.

18. Natesan S, Baer DG, Walters TJ, Babu M, Christy RJ: Adipose-derived stem cell delivery into collagen gels using chitosan microspheres. Tissue Eng Part A. 2010; 16: 136984.

19. Jeong B, Bae YH, Kim SW: In situ gelation of PEG-PLGAPEG triblock copolymer aqueous solutions and degradation thereof. J Biomed Mater Res. 2000; 50: 171-7.

20. Jeong B, Bae YH, Kim SW: Drug release from biodegradable injectable thermosensitive hydrogel of PEG-PLGA-PEG triblock copolymers. J Control Release. 2000; 63: 155-63.

21. Lee JY, Paik SY, Yuk SH, Lee JH, Ghil SH, Lee SS: The IsoIation and Characterization of Muscle Derived Stem Cells from Gastrocnemius Muscle of Rats Using the Modified Preplate Method. Korean J Urol. 2004; 45: 1279-84.

22. Deans RJ, Moseley AB: Mesenchymal stem cells: biology and potential clinical uses. Exp Hematol. 2000; 28: 875-84.

23. Levy YS, Bahat-Stroomza M, Barzilay R, Burshtein A, Bulvik $S$, Barhum Y, et al.: Regenerative effect of neural-induced human mesenchymal stromal cells in rat models of Parkinson's disease. Cytotherapy. 2008; 10: 340-52.

Correspondence address: Dr. Sae Woong Kim Department of Urology, Seoul St.Mary's Hospital 505, Banpodong, Seocho-gu Seoul 137-701, Korea FAX: +82 2 599-7839

E-mail: ksw1227@catholic.ac.kr 